\title{
SOME EXPANSIONS IN SERIES OF BESSEL FUNCTIONS*
}

\author{
BY \\ H. M. SRIVASTAVA ${ }^{1}$ (University of Victoria, Victoria, British Columbia, Canada) \\ AND \\ R. M. SHRESHTHA (Tribhuvan University, Kirtipur, Kathmandu, Nepal)
}

\begin{abstract}
A general theorem on generating functions is applied to derive a number of interesting expansions for the generalized hypergeometric ${ }_{r} F_{s}$ function in series of Bessel functions. Several further expansion formulas, relevant to the present discussion, are also considered. Many of these expansions in series of Bessel functions stem from (or are motivated by) their applicability in various seemingly diverse fields of applied sciences and engineering. With this point in view, some examples illustrating possible applications of these results are provided.
\end{abstract}

1. Introduction. In the usual notation, let $I_{\nu}(z)$ defined by

$$
I_{\nu}(z)=\sum_{n=0}^{\infty} \frac{\left(\frac{1}{2} z\right)^{\nu+2 n}}{n ! \Gamma(\nu+n+1)} \quad(|z|<\infty)
$$

denote the modified Bessel function of the first kind of order $\nu$ (cf., e.g., Watson [12, p. 77]). Expansions of various hypergeometric functions of one and more variables in series of $I_{\nu}(z)$ are scattered in the literature. Recently, Shreshtha [5] gave two such expansions for the confluent hypergeometric ${ }_{1} F_{1}$ function and extended them further to hold true for the generalized hypergeometric ${ }_{r} F_{s}$ function with $r$ numerator and $s$ denominator parameters. We recall here the most general result in Shreshtha's paper in the form (cf. [5, p. 296, Equation (3.2)]):

$$
\begin{aligned}
\left.\left(\frac{1}{4} z\right)^{\lambda}{ }_{r} F_{s}\left[\begin{array}{l}
\left(a_{r}\right) ; \\
\left(b_{s}\right) ;
\end{array} \frac{w z}{m}\right)^{m}\right]= & \frac{\Gamma(\lambda)}{\Gamma(2 \lambda)} e^{z / 2} \sum_{n=0}^{\infty}(-1)^{n} \frac{(\lambda+n) \Gamma(2 \lambda+n)}{n !} I_{\lambda+n}\left(\frac{1}{2} z\right) \\
& \cdot 2 m+r F_{m+s}\left[\begin{array}{r}
\Delta(m ;-n), \Delta(m ; 2 \lambda+n),\left(a_{r}\right) ; \\
w^{m} \\
\Delta\left(m ; \lambda+\frac{1}{2}\right),\left(b_{s}\right) ;
\end{array}\right],
\end{aligned}
$$

\footnotetext{
${ }^{*}$ Received February 10, 1987.

${ }^{1}$ The work of this author was supported, in part, by the Natural Sciences and Engineering Research Council of Canada under Grant A-7353
} 
where $\lambda \neq 0,-1,-2, \ldots ;\left(a_{r}\right)$ abbreviates the array of $r$ parameters $a_{1}, \ldots, a_{r}$, with similar interpretations for $\left(b_{s}\right)$, et cetera, and the symbol $\Delta(m ; \lambda)$ represents the array of $m$ parameters

$$
(\lambda+j-1) / m, \quad j=1, \ldots, m,
$$

$m$ being a positive integer. Throughout this paper it should indeed be understood that exceptional parameter values that would render either side of an expansion formula meaningless or undefined are tacitly excluded. Thus, for example, the parameters $\lambda+\frac{1}{2}$ and $b_{1}, \ldots, b_{s}$ in the expansion formula (1.2) are assumed to be neither zero nor a negative integer.

The special case of (1.2) when $m=1$ is a well-known result recorded, for example, by Luke [2, p. 20, Equation (4)]. A direct proof of this special case was presented by Srivastava [6] who made use of the elementary expansion:

$$
\left(\frac{1}{2} z\right)^{\lambda}=\frac{\Gamma(\lambda)}{\Gamma(2 \lambda)} e^{z} \sum_{n=0}^{\infty}(-1)^{n} \frac{(\lambda+n) \Gamma(2 \lambda+n)}{n !} I_{\lambda+n}(z),
$$

where, as also in (1.2), $\lambda \neq 0,-1,-2, \ldots$. The object of the present paper is to show that much more general expansions than (1.2) can be deduced by appropriately applying a theorem on generating functions. We also derive several further results analogous to (1.2). The expansions (3.2), (3.5), (3.6), and (3.8) in series of Bessel functions are believed to be new. Some indication of applicability of these general classes of expansion formulas in various physical situations is also provided.

2. Applications of a theorem on generating functions. We begin by recalling the following

Theorem (Srivastava and Panda [11, p. 472, Theorem 2]). Corresponding to the given sequences $\left\{\Lambda_{n}\right\}_{n=0}^{\infty}$ and $\left\{\Omega_{n}\right\}_{n=0}^{\infty}$, let

$$
P_{n}^{(\lambda)}(x ; m)=\sum_{k=0}^{[n / m]} \frac{(-n)_{m k}(\lambda+n)_{m k}}{k !} \Lambda_{k} x^{k}
$$

and

$$
\Theta_{n}(t)=\sum_{r=0}^{\infty} \frac{\Omega_{n+r}}{(\lambda+2 n+1)_{r}} \frac{t^{r}}{r !}, \quad|t|<T_{0},
$$

where $(\lambda)_{n}=\Gamma(\lambda+n) / \Gamma(\lambda), m$ is an arbitrary positive integer, and the complex parameter $\lambda$ is neither zero nor a negative integer. Suppose also that $G(z)$ is defined by

$$
G(z)=\sum_{n=0}^{\infty} \Lambda_{n} \Omega_{m n} \frac{z^{n}}{n !}, \quad|z|<S_{0} .
$$

Then

$$
\sum_{n=0}^{\infty} \frac{(-t)^{n}}{n !(\lambda+n)_{n}} P_{n}^{(\lambda)}(x ; m) \Theta_{n}(t)=G\left(x t^{m}\right),
$$

provided that $\Lambda_{0} \Omega_{0} \neq 0,\left|x t^{m}\right|<S_{0},|t|<T_{0}$, and the series on the left has a meaning. 
Setting

$$
\Lambda_{n}=\frac{\prod_{j=1}^{r}\left(a_{j}\right)_{n} \prod_{j=1}^{v}\left(d_{j}\right)_{m n}}{\prod_{j=1}^{s}\left(b_{j}\right)_{n} \prod_{j=1}^{u}\left(c_{j}\right)_{m n}} \quad \text { and } \quad \Omega_{n}=\frac{\prod_{j=1}^{p}\left(\alpha_{j}\right)_{n} \prod_{j=1}^{u}\left(c_{j}\right)_{n}}{\prod_{j=1}^{q}\left(\beta_{j}\right)_{n} \prod_{j=1}^{v}\left(d_{j}\right)_{n}}
$$

and making use of the identity:

$$
(\lambda)_{m n}=m^{m n} \prod_{j=1}^{m}\left(\frac{\lambda+j-1}{m}\right)_{n} \quad(m=1,2,3, \ldots),
$$

we find from the assertion (2.4) that (cf. [7, p. 304, Equation (3.12)])

$$
\begin{gathered}
m p+r F_{m q+s}\left[\begin{array}{l}
\left.\Delta\left[m ;\left(\alpha_{p}\right)\right],\left(a_{r}\right) ; x t^{m} m^{m(p-q)}\right] \\
\Delta\left[m ;\left(\beta_{q}\right)\right],\left(b_{s}\right) ;
\end{array}\right] \\
=\sum_{n=0}^{\infty} \frac{(-t)^{n}}{n !(\lambda+n)_{n}} \Gamma_{n}\left[\left(\alpha_{p}\right),\left(c_{u}\right) ;\left(\beta_{q}\right),\left(d_{v}\right)\right]_{p+u} F_{1+q+v}\left[\begin{array}{r}
\left(\alpha_{p}\right)+n,\left(c_{u}\right)+n ; \\
\lambda+2 n+1,\left(\beta_{q}\right)+n,\left(d_{v}\right)+n ;
\end{array}\right] \\
\cdot m(2+v)+r \\
F_{m u+s}\left[\begin{array}{c}
\Delta(m ;-n), \Delta(m ; \lambda+n), \Delta\left[m ;\left(d_{v}\right)\right],\left(a_{r}\right) ; \\
\left.x m^{m(2-u+v)}\right],(2.7)
\end{array}\right]
\end{gathered}
$$

where $\Delta\left[m ;\left(\alpha_{p}\right)\right]$ represents the array of $m p$ parameters

$$
\left(\alpha_{i}+j-1\right) / m, \quad i=1, \ldots, p \quad \text { and } j=1, \ldots, m,
$$

with similar interpretations for $\Delta\left[m ;\left(\beta_{q}\right)\right]$, et cetera, and (for convenience)

$$
\Gamma_{n}\left[\left(\alpha_{p}\right),\left(c_{u}\right) ;\left(\beta_{q}\right),\left(d_{v}\right)\right]=\frac{\prod_{j=1}^{p}\left(\alpha_{j}\right)_{n} \prod_{j=1}^{u}\left(c_{j}\right)_{n}}{\prod_{j=1}^{q}\left(\beta_{j}\right)_{n} \prod_{j=1}^{v}\left(d_{j}\right)_{n}}, \quad n \geq 0 .
$$

Now recall the relationship ( $c f .[2$, p. 22 , Equation (2)]):

$$
I_{\lambda}(z)=\frac{\left(\frac{1}{2} z\right)^{\lambda}}{\Gamma(\lambda+1)} e^{ \pm z} F_{1}\left[\begin{array}{c}
\lambda+\frac{1}{2} ; \\
2 \lambda+1 ;
\end{array} 2 z\right] \text {, }
$$

where, as usual, both upper signs or both lower signs are taken simultaneously. In view of (2.9), the expansion formula (1.2) can easily be rewritten in its equivalent form:

$$
{ }_{r} F_{s}\left[\begin{array}{l}
\left(a_{r}\right) ; \\
\left(b_{s}\right) ;
\end{array}\right.
$$

where we have also made use of the identity (2.6) with $m=2$.

Obviously, the expansion formula (1.2) or (2.10) is a very special case of $(2.7)$, with $\lambda$ replaced by $2 \lambda$, when

$$
p=q=0, \quad u-1=v=0, \quad c_{1}=\lambda+\frac{1}{2}, \quad t=z, \quad \text { and } \quad x=(w / m)^{m} .
$$


3. Further expansions and applications. Making use of the relationship ( $c f .[12, \mathrm{p}$. 147, Equation (1)]):

$$
I_{\lambda}(z) I_{\mu}(z)=\frac{\left(\frac{1}{2} z\right)^{\lambda+\mu}}{\Gamma(\lambda+1) \Gamma(\mu+1)}{ }_{2} F_{3}\left[\begin{array}{c}
\Delta(2 ; \lambda+\mu+1) ; \\
z^{2} \\
\lambda+1, \mu+1, \lambda+\mu+1 ;
\end{array}\right],
$$

we find from our expansion formula (2.7) with $\lambda$ replaced by $\lambda+\mu$, and with

$$
\begin{cases}p=q=0, & u=v=2, \quad u_{1}=\frac{1}{2}(\lambda+\mu+1), \quad u_{2}=\frac{1}{2}(\lambda+\mu+2) \\ v_{1}=\lambda+1, & v_{2}=\mu+1, \quad t=z^{2}, \quad \text { and } \quad x=(w / m)^{2 m}\end{cases}
$$

that

$$
\begin{array}{r}
\left(\frac{1}{2} z\right)^{\lambda+\mu}{ }_{r} F_{s}\left[\begin{array}{c}
\left(a_{r}\right) ; \\
\left(b_{s}\right) ;
\end{array}\left(\frac{w z}{m}\right)^{2 m}\right] \\
=\frac{\Gamma(\lambda+1) \Gamma(\mu+1)}{\lambda+\mu} \sum_{n=0}^{\infty}(-1)^{n} \frac{(\lambda+\mu+2 n)(\lambda+\mu)_{n}}{n !} I_{\lambda+n}(z) I_{\mu+n}(z) \\
{ }_{4 m+r} F_{2 m+s}\left[\begin{array}{c}
\Delta(m ;-n), \Delta(m ; \lambda+\mu+n), \Delta(m ; \lambda+1), \Delta(m ; \mu+1),\left(a_{r}\right) ; w^{2 m} \\
\Delta\left(m ; \frac{1}{2} \lambda+\frac{1}{2} \mu+\frac{1}{2}\right), \Delta\left(m ; \frac{1}{2} \lambda+\frac{1}{2} \mu+1\right),\left(b_{s}\right) ;
\end{array}\right],
\end{array}
$$

whose special case when $m=1$ is known (cf., e.g., [4, p. 224, Equation (4)]).

The expansion formula (3.2) simplifies considerably in its special case when $\lambda=\mu$. An expansion analogous to (3.2), but in series of the products

$$
I_{\lambda+2 n}(z) J_{\lambda+2 n}(z),
$$

where [cf. Equation (1.1)]

$$
J_{\nu}(z)=\sum_{n=0}^{\infty} \frac{(-1)^{n}\left(\frac{1}{2} z\right)^{\nu+2 n}}{n ! \Gamma(\nu+n+1)} \quad(|z|<\infty)
$$

denotes the (ordinary) Bessel function of order $\nu$, would also follow from the general result (2.7), since [2, p. 25, Equation (20)]

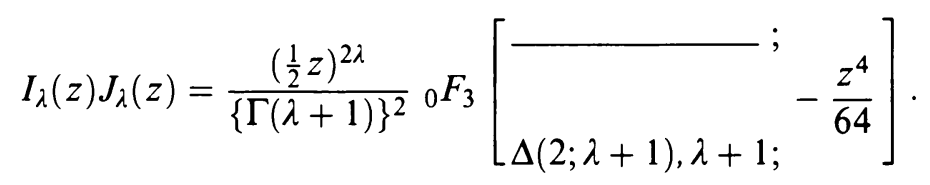

Thus, if in (2.7) we set

$$
\begin{array}{ll}
p=q=0, \quad u=v-2=0, \quad d_{1}=\frac{1}{2}(\lambda+1), \quad d_{2}=\frac{1}{2} \lambda+1, \quad t=-\frac{z^{4}}{64} \\
\text { and } \quad x=(w / m)^{4 m},
\end{array}
$$


we find that

$$
\begin{gathered}
\left(\frac{1}{2} z\right)^{2 \lambda} F_{s}\left[\begin{array}{c}
\left(a_{r}\right) ; \\
\left(b_{s}\right) ;
\end{array}\right. \\
\quad=\Gamma(\lambda+1) \sum_{n=0}^{\infty} \frac{(\lambda+2 n) \Gamma(\lambda+n)}{n !} I_{\lambda+2 n}(z) J_{\lambda+2 n}(z) \\
\cdot{ }_{4 m+r} F_{s}\left[\begin{array}{c}
\left.\Delta(m ;-n), \Delta(m ; \lambda+n), \Delta\left(m ; \frac{1}{2} \lambda+\frac{1}{2}\right), \Delta\left(m ; \frac{1}{2} \lambda+1\right),\left(a_{r}\right) ; w^{4 m}\right], \\
\left(b_{s}\right) ;
\end{array}\right],
\end{gathered}
$$

whose special case when $m=1$ does not seem to have been recorded earlier.

Yet another expansion in series of Bessel functions would result from (2.7) if we set

$$
p=q=u=v=0, \quad t=\left(\frac{1}{2} z\right)^{2}, \quad \text { and } \quad x=(w / m)^{2 m},
$$

and apply the definition (1.1). We thus obtain

$$
\begin{aligned}
\left.\left(\frac{1}{2} z\right)^{\lambda}{ }_{r} F_{s}\left[\begin{array}{l}
\left(a_{r}\right) ; \\
\left(b_{s}\right) ;
\end{array} \frac{w z}{2 m}\right)^{2 m}\right]= & \sum_{n=0}^{\infty}(-1)^{n} \frac{(\lambda+2 n) \Gamma(\lambda+n)}{n !} I_{\lambda+2 n}(z) \\
& { }_{2 m+r} F_{s}\left[\begin{array}{c}
\left.\Delta(m ;-n), \Delta(m ; \lambda+n),\left(a_{r}\right) ; w^{2 m}\right], \\
\left(b_{s}\right) ;
\end{array}\right]
\end{aligned}
$$

whose special case when $m=1$ yields a known result (cf.,e.g., [4, p. 223, Equation (1)]).

Next we deduce a confluent case of the expansion formula (2.7) when $t$ is replaced by $\lambda t$, and $x$ by $x / \lambda^{m}$, and $\lambda \rightarrow \infty$. Thus we have (cf. [7, p. 305, Equation (3.13)])

$$
\begin{array}{r}
m_{m p+r} F_{m q+s}\left[\begin{array}{l}
\left.\Delta\left[m ;\left(\alpha_{p}\right)\right],\left(a_{r}\right) ; x t^{m} m^{m(p-q)}\right] \\
\Delta\left[m ;\left(\beta_{q}\right)\right],\left(b_{s}\right) ;
\end{array}\right] \\
=\sum_{n=0}^{\infty} \frac{(-t)^{n}}{n !} \Gamma_{n}\left[\left(\alpha_{p}\right),\left(c_{u}\right) ;\left(\beta_{q}\right),\left(d_{v}\right)\right]_{p+u} F_{q+v}\left[\begin{array}{c}
\left(\alpha_{p}\right)+n,\left(c_{u}\right)+n ; t \\
\left(\beta_{q}\right)+n,\left(d_{v}\right)+n ;
\end{array}\right] \\
{ }_{m(1+v)+r} F_{m u+s}\left[\begin{array}{c}
\Delta(m ;-n), \Delta\left[m ;\left(d_{v}\right)\right],\left(a_{r}\right) ; x m^{m(1-u+v)} \\
\Delta\left[m ;\left(c_{u}\right)\right],\left(b_{s}\right) ;
\end{array}\right],
\end{array}
$$

which indeed would follow also by applying another result given by Srivastava and Panda [11, p. 468, Theorem 1], using (2.5) and (2.6).

If in (3.7) we set

$$
p=q=0, \quad u=v-1=0, \quad d_{1}=\lambda+1, \quad t=\left(\frac{1}{2} z\right)^{2}, \quad \text { and } \quad x=(w / m)^{2 m},
$$


and make use of the definition (1.1), we shall obtain the expansion formula:

$$
\begin{aligned}
& \left(\frac{1}{2} z\right)^{\lambda}{ }_{r} F_{s}\left[\begin{array}{l}
\left(a_{r}\right) ; \\
\left(\frac{w z}{2 m}\right)^{2 m}
\end{array}\right] \\
& =\Gamma(\lambda+1) \sum_{n=0}^{\infty} \frac{\left(-\frac{1}{2} z\right)^{n}}{n !} I_{\lambda+n}(z) 2 m+r F_{s}\left[\begin{array}{c}
\Delta(m ;-n), \Delta(m ; \lambda+1),\left(a_{r}\right) ; \\
\left.w^{2 m}\right) ;
\end{array}\right] \text {, }
\end{aligned}
$$

which, when $m=1$, yields a known result (cf., e.g., [4, p. 224, Equation (2)]).

Finally, we give a mild generalization of the expansion formula (3.7). Incidentally, this generalization of (3.7), given by Equation (3.9) below, is not contained in (2.7). Indeed, it would follow readily if we apply yet another result of Srivastava and Panda [11, p. 472, Theorem 3], again using (2.5) and (2.6). We thus find that (cf. [7, p. 305, Equation (3.14)])

$$
\begin{gathered}
m_{m p+r} F_{m q+s}\left[\begin{array}{l}
\left.\Delta\left[m ;\left(\alpha_{p}\right)\right],\left(a_{r}\right) ; x t^{m} m^{m(p-q)}\right] \\
\Delta\left[m ;\left(\beta_{q}\right)\right],\left(b_{s}\right) ;
\end{array}\right. \\
=\beta \sum_{n=0}^{\infty} \frac{(-t)^{n}}{n !}(1-\alpha n+\beta)_{n-1} \Gamma_{n}\left[\left(\alpha_{p}\right),\left(c_{u}\right) ;\left(\beta_{q}\right),\left(d_{v}\right)\right] \\
\cdot 1+p+u F_{q+v}\left[\begin{array}{c}
(1-\alpha) n+\beta,\left(\alpha_{p}\right)+n,\left(c_{u}\right)+n ; \\
\left(\beta_{q}\right)+n,\left(d_{v}\right)+n ;
\end{array}\right] \\
\left.\begin{array}{c}
\Delta(m ;-n), \Delta(m ; 1+\beta /(1-\alpha)), \Delta\left[m ;\left(d_{v}\right)\right],\left(a_{r}\right) ; \\
x(m ; \beta /(1-\alpha)), \Delta(m ; 1-\alpha n+\beta), \Delta\left[m ;\left(c_{u}\right)\right],\left(b_{s}\right) ;
\end{array}\right]
\end{gathered}
$$

which, for $\alpha=0$, corresponds essentially to the expansion formula (3.7).

For numerous further applications of the special cases of the expansion formulas (2.7) and (3.7) when $m=1$, see Erdélyi et al. [1, Chapter 7], Luke ([2, Chapters 1 and 7], [3, Chapter 9], [4, Chapter 5]), and Watson [12, Chapters 5 and 11]. As a matter of fact, each of the expansions (2.7), (3.7), and (3.9), as well as their various multivariable extensions, have been considered rather systematically in the literature (see, for example, [7], [8], [9, Chapter 9], and [10]).

It is fairly well known that hypergeometric series (and hypergeometric polynomials) in one and more variables are encountered rather frequently in a wide variety of problems in applied mathematics and theoretical physics, and indeed also in engineering sciences, statistics, and operations research (see, for examples, [9, Section 1.7] and the various references cited there). For instance, a considerably vast field of physical and quantum chemical situations lead naturally to such special cases of the 
hypergeometric polynomials

$$
\Phi_{n, m}^{(\lambda)}(x)={ }_{2 m+r} F_{s}\left[\begin{array}{r}
\Delta(m ;-n), \Delta(m ; \lambda+n),\left(a_{r}\right) ; \\
x \\
\left(b_{s}\right) ;
\end{array}\right]
$$

and

$$
\Psi_{n, m}(x)={ }_{m+r} F_{s}\left[\begin{array}{r}
\Delta(m ;-n),\left(a_{r}\right) ; \\
x \\
\left(b_{s}\right) ;
\end{array}\right],
$$

occurring in our expansion formulas, as the Bessel polynomials and the classical orthogonal polynomials including, for example, Hermite, Jacobi (and, of course, Gegenbauer, Legendre, and Tchebycheff), and Laguerre polynomials; of these the Hermite polynomials:

$$
\begin{aligned}
H_{n}(x) & =\sum_{k=0}^{[n / 2]} \frac{(-1)^{k} n !}{k !(n-2 k) !}(2 x)^{n-2 k} \\
& =(2 x)^{n}{ }_{2} F_{0}\left[\begin{array}{r}
\Delta(2 ;-n) ; \\
-\frac{1}{x^{2}}
\end{array}\right]
\end{aligned}
$$

play an important rôle in problems involving Laplace's equation in parabolic coordinates, in various problems in quantum mechanics, and in probability theory. Indeed the Hermite polynomials $H_{n}(x)$, defined by $(3.12)$, correspond to the hypergeometric polynomials $(3.11)$ when

$$
m=2 \text { and } r=s=0,
$$

and arise, in particular, in the investigation of Schrödinger's equation for a harmonic oscillator (cf., e.g., [13, Chapter 5]).

Suppose now that, in a certain boundary value problem in potential theory, such as in the familiar Dirichlet problem for a parabolic cylinder, we are required to express a given real-valued function $f(x)$, defined on the interval $(-\infty, \infty)$ and (at least) piecewise smooth on every finite closed subinterval $[-\delta, \delta], \delta>0$, as the FourierHermite series:

$$
f(x)=\sum_{n=0}^{\infty} \omega_{n} H_{n}(x), \quad-\infty<x<\infty .
$$

In every such situation in which $f(x)$ is of hypergeometric type, the unknown coefficients $\omega_{n}$ can indeed be determined by merely comparing each member of (3.13) with the corresponding member of an appropriate special case $m=2$ of one or the other of the expansion formulas derived in this section.

Finally, since the formulas presented in this paper express a generalized hypergeometric function in series of the Bessel function $I_{\nu}(z)$ or $J_{\nu}(z)$, or their various products, our results will naturally apply also in numerous other boundary value problems (such as those in astrophysics, electromagnetic theory, and the theory of heat conduction) requiring these general classes of series expansions of Neumann and Neumann-Gegenbauer types (cf. [12, Chapter 16]). 


\section{REFERENCES}

[1] A. Erdélyi, W. Magnus, F. Oberhettinger, and F. G. Tricomi, Higher Transcendental Functions, Vol. II, McGraw-Hill, New York, Toronto and London, 1953

[2] Y. L. Luke, Integrals of Bessel Functions, McGraw-Hill, New York, Toronto and London, 1962

[3] Y. L. Luke, The Special Functions and Their Approximations, Vol. II, Academic Press, New York and London, 1969

[4] Y. L. Luke, Mathematical Functions and Their Approximations, Academic Press, New York, San Francisco and London, 1975

[5] R. M. Shreshtha, Expansions in series of Bessel functions, C. R. Acad. Bulgare Sci. 35, 295-297 (1982)

[6] H. M. Srivastava, Some expansions of generalized Whittaker functions, Proc. Cambridge Philos. Soc. 61, 895-896 (1965)

[7] H. M. Srivastava, Some polynomial expansions for functions of several variables, IMA J. Appl. Math. 27, 299-306 (1981)

[8] H. M. Srivastava and M. C. Daoust, Certain generalized Neumann expansions associated with the Kampé de Fériet function, Nederl. Akad. Wetensch. Indag. Math. 31, 449- 457 (1969)

[9] H. M. Srivastava and P. W. Karlsson, Multiple Gaussian Hypergeometric Series, Halsted Press (Ellis Horwood Limited, Chichester), John Wiley and Sons, New York, Chichester, Brisbane and Toronto, 1985

[10] H. M. Srivastava and R. Panda, Expansion theorems for the $H$ function of several complex variables, J. Reine Angew. Math. 288, 129-145 (1976)

[11] H. M. Srivastava and R. Panda, A note on certain results involving a general class of polynomials, Boll. Un. Mat. Ital. A (5) 16, 467- 474 (1979)

[12] G. N. Watson, A Treatise on the Theory of Bessel Functions, Second ed., Cambridge University Press, Cambridge, London and New York, 1966

[13] A. Yariv, An Introduction to Theory and Applications of Quantum Mechanics, John Wiley and Sons, New York, Chichester, Brisbane, Toronto and Singapore, 1982 